\title{
Prodrug Systems (II): A Perspective of Polymer-Based Doxorubicin Prodrug Systems towards Chemotherapy
}

\author{
Zhao Wang, ${ }^{a}$ Filipe Olim, ${ }^{b}$ Jingjing Sun, ${ }^{c}$ Ana Rute Neves, ${ }^{b}$ Fátima Mendes, ${ }^{b}$ Helena Tomás, ${ }^{, b}$ \\ and Ruilong Sheng ${ }^{\star, b}$ \\ a School of Material Engineering, Jinling Institute of Technology, Nanjing, Jiangsu 211169, China \\ ${ }^{b}$ CQM - Centro de Química da Madeira, Universidade da Madeira, Campus da Penteada, \\ 9000-390 Funchal, Portugal \\ ${ }^{c}$ Center for Pharmacogenetics, Department of Pharmaceutical Sciences, School of Pharmacy, \\ University of Pittsburgh, Pittsburgh, PA 15261, United States
}

Email: ruilong.sheng@staff.uma.pt (R. S.)

\begin{abstract}
Utilizing biocompatible polymers as platforms to covalently conjugate with chemotherapeutics to construct polymer-based prodrugs and their nano drug delivery systems has attracted great attention in recent years. This perspective reviewed state-of-the-arts for polymer-based doxorubicin prodrugs and the related nanodelivery systems, including: (1) $\mathrm{pH}$-responsive polymer-doxorubicin prodrugs/conjugates; (2) $\mathrm{pH} / \mathrm{redox}$ dual responsive prodrugs/conjugates; (3) reactive oxygen species/hypoxia-responsive polymer-doxorubicin prodrugs; (4) tumor receptor targeting polymer prodrugs; (5) enzyme-responsive polymer-doxorubicin prodrugs. Finally, possible future perspectives were also stated and discussed.
\end{abstract}

Keywords polymers, doxorubicin, prodrug, stimuli-responsive, drug delivery, nanosystem

Developing prodrugs as chemotherapeutic agents is a rapid growing and promising research field. Efficient prodrugs or conjugates need to meet the requirements of low serum protein binding, high stability in blood circulation, reticuloendothelial system (RES)-mediated immune clearance, tumor accumulation and high cellular internalization. ${ }^{[1]}$ To achieve high drug delivery efficiency, it is essential to design prodrugs or conjugates with good water solubility, controllable structures and molecular weight, tunable nanoaggregate architecture, as well as enhanced drug loading/encapsulation capacity. ${ }^{[1,2]}$

In general, prodrug-based chemotherapeutic system could be divided into two categories: small molecular prodrugs and polymer-based prodrugs. Compared to small molecular prodrug counterparts, ${ }^{[3]}$ polymer-based prodrugs exhibit higher stability, prolonged blood circulation time, higher drug loading efficiency, enhanced cellular uptake, increased bioavailability, and so on. Most studied polymer-based prodrugs are polymer-doxorubicin (Dox) conjugates. Some tumor-related factors, such as low $\mathrm{pH}$, bioreductive substances GSH, reactive oxygen species (ROS), as well as specific protease, could be used to trigger prodrug release through specific biochemical reactions and then improve the chemotherapeutic performance. Accordingly, smart and stimuli-responsive polymer-doxorubicin prodrugs were developed as these following types: (1) $\mathrm{pH}$-responsive polymeroxorubicin conjugates, such as: acid-labile cis-aconityl containing mPEG- $b$-PAE-cis-DOX prodrug, ${ }^{[4]}$ SPAAC-clickonjugates mPEG- $b$-PLA-g-DOX, ${ }^{[5]} \mathrm{pH}$-induced charge conversion prodrug PLL (CB/DOX)- $b$-PMPC, ${ }^{[6]}$ cyclodextrinased supramolecular prodrug PRMO@DOX, ${ }^{[7]}$ pluronic $\mathrm{pH}$ sensitive prodrug P123-CAD/F127-PBA, ${ }^{[8]}$ with these prodrugs normally containing $\mathrm{pH}$-sensitive $(4.5-6.5)$ linkage bonds including Schiff-base, hydrozone and aconityl; (2) $\mathrm{pH} /$ redoxdual responsive prodrugs/conjugates, such as: hyperbranched polyglycerol (hPG)-based NG-DOX conjugates, ${ }^{[9]}$ EG2K-SS-CBA-DOX prodrug, ${ }^{[10]} \mathrm{pH} / \mathrm{GSH}$-sensitive prodrug
PDPAO@imine-DOX/cis-6MP, ${ }^{[11]}$ cross-linked dendritic MPEGb-PAMAM-LA/DOX prodrug ${ }^{[12]}$ and $\mathrm{pH} /$ bioreduction-dual $^{2}$ responsive [PEEP- $b$-(PBYP-hyd-DOX)-Se] $]_{2}$ prodrug, ${ }^{[13]}$ these prodrugs having $\mathrm{pH}$-sensitive moieties and redox-sensitive moieties (disulfide/diselenide linkages) in certain positions; (3) reactive oxygen species (ROS)/hypoxia-responsive polymerdoxorubicin prodrugs, such as ROS/PDT-dual functional prodrug nanosystems PEG-TK-DOX@pheophorbide A (PhA $)^{[14]}$ and Ce6@PPE-TK-DOX, ${ }^{[15]}$ in which thioketal acts as the ROS-cleavable linker; (4) tumor receptor targeting polymerdoxorubicin prodrugs, such as: FA-P(MPC-co-PEGMA-BZ)- $g$ DOX prodrug (targeting: Folate receptor) ${ }^{[16]}$ and TPGS- $\mathrm{CH}=\mathrm{N}$ DOX/DSPE-PEG-CRGD (targeting: intergrin), ${ }^{[17]}$ whose biotargeting effect greatly depends on the density of targeting moieties (optimum: 5\%-10\% molar ratio); and (5) enzymeresponsive polymer-doxorubicin prodrugs, such as $\mathrm{pH} /$ legumain enzyme-dual responsive prodrug-carbon dot CDs-C9-AANL-DOX. ${ }^{[18]}$ The related study is relatively rare due to the synthesis bottleneck of enzyme-cleavable peptide linkers. Besides, there are some multi-stimuli (photo/redox-dual

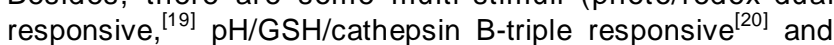
$\mathrm{pH} /$ redox/AIEgen prodrug theranostic ${ }^{[21]}$ ) systems that have been newly developed. These promising works showed that the smart and stimuli-responsive polymer-doxorubicin prodrugs could be employed as potential chemotherapeutic systems. However, the therapeutic efficiency of single-component prodrug is greatly limited due to several factors such as disease complexity, multidrug resistance (MDR) and off-targeting effect. $^{[22]}$ To overcome these disadvantages, polymer-doxorubicin-based co-delivery system such as CAD-PLGA-PEGPLGA-CAD-doxorubicin/docetaxel for in situ synergistic chemotherapy, ${ }^{[23]}$ has been developed. Compared to multidrug co-delivery systems, only few biocompatible polymer-based doxorubicin/gene co-delivery (e.g., dox-P53DNA co-delivery $\mathrm{mPEG}$ $b$-PBYP-hyd-DOX/mPEG-b-PBYP-g-DAE nanocarrier $)^{[24]}$ 
Scheme 1 Various types of polymer-based doxorubicin conjugates/prodrug systems
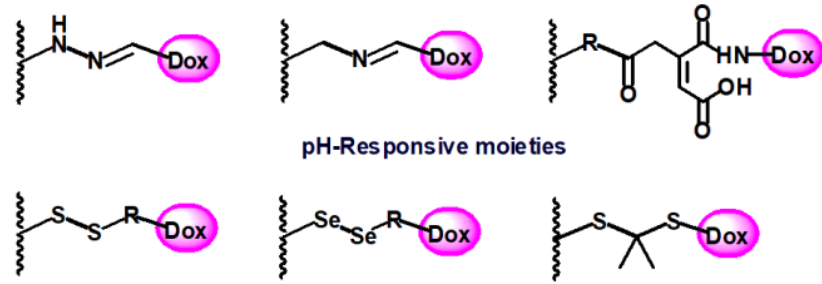

Bioreduction-Responsive moieties

ROS-Res ponsive moiety

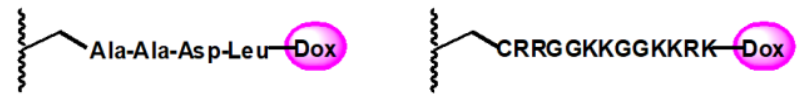

Enzyme-Responsive moieties
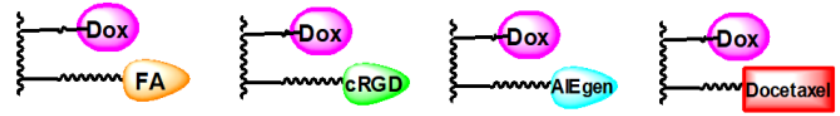

Biotargeting moieties

Bioimaging moiety Drug co-delivery

systems have been developed. Up to date, creating a doxorubicin/gene programmable co-delivery system to achieve synergistic effect, overcome MDR and inhibit the anti-apoptotic process is still a not-fully developed area. The polymer-based doxorubicin prodrugs are shown in Scheme 1.

For the future outlook of polymer-based doxorubicin prodrug chemotherapeutic systems, we can expect the research and development $(R \& D)$ in the following areas: (1) using natural-based sustainable resources, ${ }^{[25]}$ such as: polysaccharides, polypeptides/peptoids and polylipids/lipoids, along with green, efficient, controllable and modular methods/ strategies to synthesize natural polymer-based doxorubicin prodrugs/conjugates; (2) expanding the structure/function diversity of the polymer-based doxorubicin prodrugs, especially "smart" (stimuli-responsive, multifunction-integrated, receptor targeting, bio-recognition, etc.) polymer-based doxorubicin prodrugs towards precision and personalized medicine; especially, it is worth integrating polymer-doxorubicin prodrugs with ${ }^{18} \mathrm{~F}$, ${ }^{64} \mathrm{Cu}$-based positron electron tomography (PET) system or functional inorganic nanoparticles ${ }^{[26]}$ for creating efficient multi-channel prodrug-based theranostics; (3) Elucidating the structure-function relationship (SFR) between the architectures of polymer-based doxorubicin prodrugs/ conjugates and their physicochemical/biological functions; (4) Based on in vitro bioevaluation data, optimizing the (natural) polymer-based doxorubicin prodrugs and their nanodelivery systems for programmable/synergistic theranostic performance (5) Artificial intelligence assisted design (AIAS) and simulation of polymer-based prodrug system towards post-information biomedicine era. It is necessary to rationally and systematically address the above-mentioned topics (Scheme 2).

\section{Acknowledgement}

Dr. Ruilong Sheng thanks FCT-Fundação para a Ciência e a Tecnologia (project UIDB/00674/2020, and Programmatic Fund -UIDP/00674/2020, CQM, Portuguese Government funds), ARDITI- Agência Regional para o Desenvolvimento da Investigação Tecnologia e Inovação, through the project M1420-01-0145- FEDER-000005-Centro de Química da Madeira-CQM ${ }^{+}$(Madeira 14-20) and ARDITI-2017-ISG-003 for sponsorship. Dr. Zhao Wang thanks the foundation support from Jinling Institute of Technology (No. jit-b-201828) and Natural Science Foundation of Jiangsu Province (No. BK20190113).
Scheme 2 Future perspective of polymer-based doxorubicin prodrugs and their nanodelivery systems

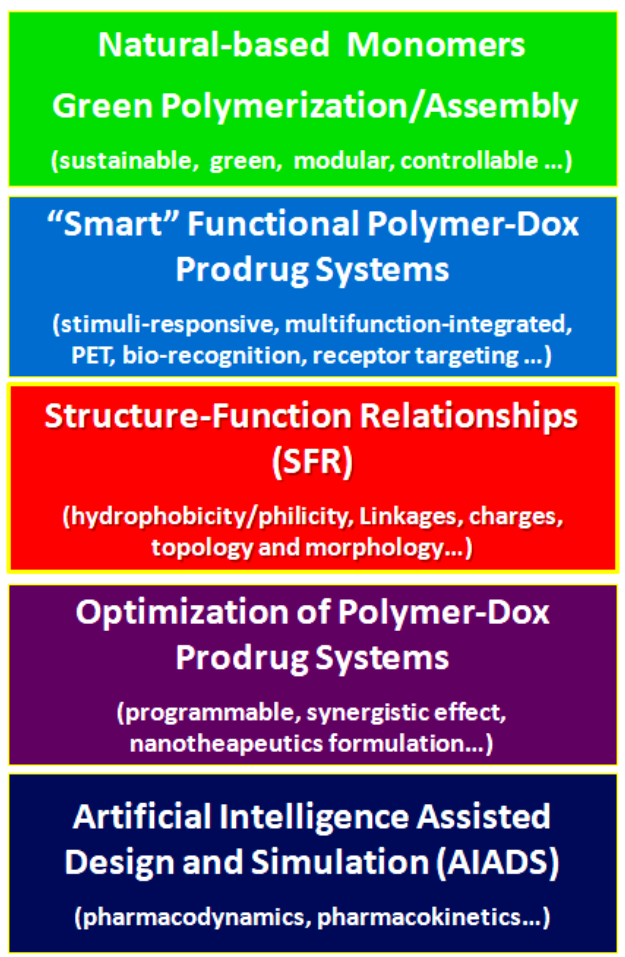

\section{Conflict of Interest}

The authors declare no conflict of interest.

Copyright (C) 2021 Zhao Wang, Filipe Olim, Jingjing Sun, Ana Rute Neves, Fátima Mendes, Helena Tomás, and Ruilong Sheng. This article is an open access article distributed under the terms and conditions of the Creative Commons Attribution (CC BY) license (http://creativecommons.org/licenses/by/4.0/). The use, distribution or reproduction in other forums is permitted, provided the original author(s) or licensor are credited and that the original publication in this journal is cited, in accordance with accepted academic practice. No use, distribution or reproduction is permitted which does not comply with these terms.

\section{References}

[1] (a) Cao, Z..; Chen, Z.; Sun, C.; Li, H.; Wang, H.; Cheng, Q.; Zuo, Z.; Wang, J.; Liu, Y.; Wang, Y.; Wang, J. Overcoming tumor resistance to cisplatin by cationic lipid-assisted prodrug nanoparticles. Biomaterials 2016, 94, 9-19; (b) Cheetham, A. G.; Chakroun, R. W.; Ma, W.; Cui, H. Self-assembling prodrugs. Chem. Soc. Rev. 2017, 46, 6638-6663.

[2] (a) Nicolas, J. Drug-Initiated Synthesis of Polymer Prodrugs: Combining Simplicity and Efficacy in Drug Delivery. Chem. Mater. 2016, 28, 1591-1606; (b) Su, H.; Zhang, W.; Wang, H.; Wang, F.; Cui, H., Paclitaxel-Promoted Supramolecular Polymerization of Peptide Conjugates. J. Am. Chem. Soc. 2019, 141, 11997-12004; (c) Cao, D.; He, J.; Xu, J.; Zhang, M.; Zhao, L.; Duan, G.; Cao, Y.; Zhou, R.; Ni, P. Polymeric prodrugs conjugated with reductionsensitive dextran-camptothecin and $\mathrm{pH}$-responsive dextrandoxorubicin: an effective combinatorial drug delivery platform for cancer therapy. Polym. Chem. 2016, 7, 4198-4212.

[3] Olim, F.; Neves, R. A.; Wang, Z.; Sun, J.; Tomás, H.; Sheng, R. Prodrug Systems (I): Lipid-Based Doxorubicin Prodrugs and Their Nanodelivery Systems. Med. Res. 2020, 4, 200013.

[4] Huang, X.; Liao, W.; Xie, Z.; Chen, D.; Zhang, C. Y., A $\mathrm{pH}$-responsive prodrug delivery system self-assembled from acid-labile doxorubicin-conjugated amphiphilic $\mathrm{pH}$-sensitive block 
copolymers. Mater. Sci. Eng. C 2018, 90, 27-37.

[5] He, J.; Wang, W.; Zhou, H.; She, P.; Zhang, P.; Cao, Y.; Zhang, X., A novel $\mathrm{pH}$-sensitive polymeric prodrug was prepared by SPAAC click chemistry for intracellular delivery of doxorubicin and evaluation of its anti-cancer activity in vitro. J. Drug Deliv. Sci. Technol. 2019, 53, 101130.

[6] Ma, B.; Zhuang, W.; Wang, Y.; Luo, R.; Wang, Y. pH-sensitive doxorubicin-conjugated prodrug micelles with charge-conversion for cancer therapy. Acta Biomater. 2018, 70, 186-196.

[7] Bai, S.; Zhang, X.; Ma, X.; Chen, J.; Chen, Q.; Shi, X.; Hou, M.; Xue, P.; Kang, Y.; Xu, Z. Acid-active supramolecular anticancer nanoparticles based on cyclodextrin polyrotaxanes damaging both mitochondria and nuclei of tumor cells. Biomater. Sci. 2018, 6, 3126-3138.

[8] Xu, C.; Xu, J.; Zheng, Y.; Fang, Q.; Lv, X.; Wang, X.; Tang, R. Active-targeting and acid-sensitive pluronic prodrug micelles for efficiently overcoming MDR in breast cancer. J. Mater. Chem. B 2020, 8, 2726-2737.

[9] Zhang, X.; Achazi, K.; Steinhilber, D.; Kratz, F.; Dernedde, J.; Haag, R. A facile approach for dual-responsive prodrug nanogels based on dendritic polyglycerols with minimal leaching. J. Controlled Release 2014, 174, 209-216.

[10] Zhang, Z.; Yu, M.; An, T.; Yang, J.; Zou, M.; Zhai, Y.; Sun, W.; Cheng, G. Tumor Microenvironment Stimuli-Responsive Polymeric Prodrug Micelles for Improved Cancer Therapy. Pharm. Res. 2019, $37,4$.

[11] Liao, J.; Peng, H.; Wei, X.; Song, Y.; Liu, C.; Li, D.; Yin, Y.; Xiong, X.; Zheng, H.; Wang, Q. A bio-responsive 6-mercaptopurine/ doxorubicin based "Click Chemistry" polymeric prodrug for cancer therapy. Mater. Sci. Eng. C 2020, 108, 110461.

[12] Zhang, Y.; Xiao, C.; Li, M.; Ding, J.; He, C.; Zhuang, X.; Chen, X., Core-cross-linked micellar nanoparticles from a linear-dendritic prodrug for dual-responsive drug delivery. Polym. Chem. 2014, 5, 2801-2808.

[13] Ma, G.; Liu, J.; He, J.; Zhang, M.; Ni, P., Dual-Responsive Polyphosphoester-Doxorubicin Prodrug Containing a Diselenide Bond: Synthesis, Characterization, and Drug Delivery. ACS Biomater. Sci. Eng. 2018, 4, 2443-2452.

[14] Kim, Y.; Uthaman, S.; Pillarisetti, S.; Noh, K.; Huh, K. M.; Park, I.-K. Bioactivatable reactive oxygen species-sensitive nanoparticulate system for chemo-photodynamic therapy. Acta Biomater. 2020, 108, 273-284.

[15] Pei, P.; Sun, C.; Tao, W.; Li, J.; Yang, X.; Wang, J., ROS-sensitive thioketal-linked polyphosphoester-doxorubicin conjugate for precise phototriggered locoregional chemotherapy. Biomaterials 2019, 188, 74-82

[16] Li, L.; Song, Y.; He, J.; Zhang, M.; Liu, J.; Ni, P., Zwitterionic shielded polymeric prodrug with folate-targeting and $\mathrm{pH}$ responsiveness for drug delivery. J. Mater. Chem. B 2019, 7, 786-795.

[17] Bao, Y.; Yin, M.; Hu, X.; Zhuang, X.; Sun, Y.; Guo, Y.; Tan, S.; Zhang, Z. A safe, simple and efficient doxorubicin prodrug hybrid micelle for overcoming tumor multidrug resistance and targeting delivery. J. Controlled Release 2016, 235, 182-194.

[18] Li, Y.; Niu, Y.; Zhu, J.; Gao, C.; Xu, Q.; He, Z.; Chen, D.; Xu, M.; Liu, $\mathrm{Y}$. Tailor-made legumain/ $\mathrm{pH}$ dual-responsive doxorubicin prodrugembedded nanoparticles for efficient anticancer drug delivery and in situ monitoring of drug release. Nanoscale 2020, 12, 2673-2685.

[19] Wu, M.; Li, J.; Lin, X.; Wei, Z.; Zhang, D.; Zhao, B.; Liu, X.; Liu, J. Reduction/photo dual-responsive polymeric prodrug nanoparticles for programmed siRNA and doxorubicin delivery. Biomater. Sci. 2018, 6, 1457-1468.

[20] Duan, Z.; Cai, H.; Zhang, H.; Chen, K.; Li, N.; Xu, Z.; Gong, Q.; Luo, K. PEGylated Multistimuli-Responsive Dendritic Prodrug-Based Nanoscale System for Enhanced Anticancer Activity. ACS Appl. Mater. Interfaces 2018, 10, 35770-35783.

[21] Su, X.; Ma, B.; Hu, J.; Yu, T.; Zhuang, W.; Yang, L.; Li, G.; Wang, Y. Dual-Responsive Doxorubicin-Conjugated Polymeric Micelles with Aggregation-Induced Emission Active Bioimaging and Charge Conversion for Cancer Therapy. Bioconjugate Chem. 2018, 29, 4050-4061.

[22] Yang, Z.; Gao, D.; Cao, Z.; Zhang, C.; Cheng, D.; Liu, J.; Shuai, X. Drug and gene co-delivery systems for cancer treatment. Biomater. Sci. 2015, 3, 1035-1049.

[23] Zhang, Y.; Zhang, J.; Xu, W.; Xiao, G.; Ding, J.; Chen, X., Tumor microenvironment-labile polymer-doxorubicin conjugate thermogel combined with docetaxel for in situ synergistic chemotherapy of hepatoma. Acta Biomater. 2018, 77, 63-73.

[24] (a) Liu, J.; He, J.; Zhang, M.; Xu, G.; Ni, P., A synergistic polyphosphoester-based co-delivery system of the anticancer drug doxorubicin and the tumor suppressor gene p53 for lung cancer therapy. J. Mater. Chem. B 2018, 6, 3262-3273; (b) Pan, J.; Rostamizadeh, K.; Filipczak, N.; Torchilin, V. P., Polymeric Co-Delivery Systems in Cancer Treatment: An Overview on Component Drugs' Dosage Ratio Effect. Molecules 2019, 24, 1035; (c) Sun, H.; Yarovoy, I.; Capeling, M.; Cheng, C. Polymers in the Co-delivery of siRNA and Anticancer Drugs for the Treatment of Drug-resistant Cancers. Topics Curr. Chem. 2017, 375, 24; (d) Kubo, S.; Takagi-Kimura, M.; Tagawa, M.; Kasahara, N. Dualvector prodrug activator gene therapy using retroviral replicating vectors. Cancer Gene Ther. 2019, 26, 128-135.

[25] Wang, Z.; Neves, R. A.; Olim, F.; Tomás, H.; Tang, S.; Sheng, R. Green Polymers toward Nanobiotechnology $(\mathrm{I})$ : Synthesis of Glycopolypeptides and Their Analogues. Gen. Chem. 2019, 5 , 190010.

[26] Xie, A.; Hanif, S.; Ouyang, J.; Tang, Z.; Kong, N.; Kim, N. Y.; Qi, B.; Patel, D.; Shi, B.; Tao, W. Stimuli-responsive prodrug-based cancer nanomedicine. EBioMedicine 2020, 56, 102821.

Received December 22, 2020

Accepted January 31, 2021 